\title{
Low-Valent Tungsten Catalysis Enables Site-Selective Isomerization- Hydroboration of Unactivated Alkenes
}

\author{
Tanner C. Jankins, ${ }^{\dagger \S}$ Raul Martin-Montero, ${ }^{\ddagger \S}$ Phillippa Cooper, ${ }^{\dagger}$ Ruben Martin, ${ }^{\ddagger £ *}$ Keary M. Engle ${ }^{\dagger *}$ \\ $\dagger$ Department of Chemistry, The Scripps Research Institute, 10550 North Torrey Pines Road, La Jolla, California, 92037, \\ United States
}

\$ Institute of Chemical Research of Catalonia (ICIQ), The Barcelona Institute of Science and Technology, Av. Països Catalans 16, 43007 Tarragona, Spain

$£$ ICREA, Passeig Lluís Companys, 23, 08010, Barcelona, Spain

Supporting Information Placeholder

ABSTRACT: A tungsten-catalyzed hydroboration of unactivated alkenes at distal $\mathrm{C}\left(s p^{3}\right)-\mathrm{H}$ bonds aided by native directing groups is described herein. The method is characterized by its simplicity, exquisite regio- and chemoselectivity and wide substrate scope, offering a complementary site-selectivity pattern to other metal-catalyzed borylation reactions and chain-walking protocols.

Chain-walking has emerged as a powerful strategy for forging $\mathrm{C}-\mathrm{C}$ bonds at remote $\mathrm{C}\left(s p^{3}\right)$ sites by controlled migration of the metal catalyst through a hydrocarbon side chain. ${ }^{1}$ At present, these protocols can promote functionalization at either the terminal position of the alkyl chain or adjacent to a stabilizing group (Scheme 1a, paths $a \& b$ ). Despite recent advances, several challenges remain to be addressed. Among them, expanding the scope of chain-walking reactions beyond $\mathrm{C}-\mathrm{C}$ bond-formation ${ }^{2}$ and achieving tunable selectivity to target previously inaccessible $\mathrm{C}\left(s p^{3}\right)-\mathrm{H}$ sites would be worthwhile endeavors for chemical innovation.

\section{Scheme 1. Olefin Functionalization via Chain-Walking.}

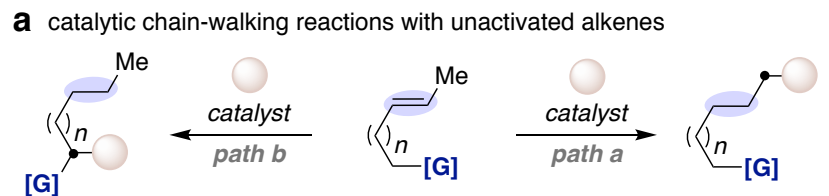

$\alpha$-selectivity $\quad[\mathrm{G}]=$ aryl, $\mathrm{BX}_{2}, \mathrm{C}(=\mathrm{O}) \mathrm{R} \quad$ linear selectivity b this work: control chain-walking site-selectivity with native DG

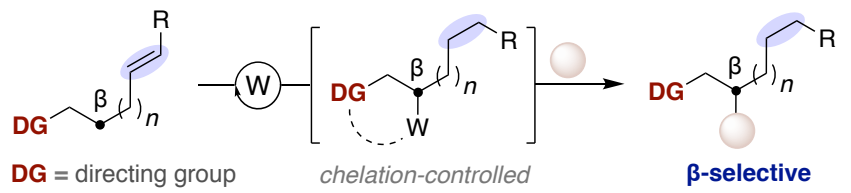

Elegant work by Chirik ${ }^{3 \mathrm{a}-\mathrm{g}}$ and others $\mathrm{e}^{3 \mathrm{e}-\mathrm{f}}$ has shown the ability of Co catalysts to trigger chain-walking borylation of unactivated alkenes for forging $\mathrm{C}\left(s p^{3}\right)-\mathrm{B}$ bonds at ter- minal primary sites or $\alpha$ - to arenes or alkyl boronates. Although Koh recently leveraged the stabilizing features of $\pi$-benzyl intermediates (path $b,[\mathrm{G}]=$ aryl) to promote a homobenzylic protoborylation, ${ }^{5}$ the means to enable $\mathrm{C}-\mathrm{B}$ bond-formation through alkylmetal stabilization at lessactivated $s p^{3}$ sites via chain-walking still remains elusive. Based on recent findings by our group, ${ }^{6}$ we wondered whether we could enable a W-catalyzed $\mathrm{C}\left(s p^{3}\right)$-B bondformation controlled by native directing groups at $\mathrm{C}\left(s p^{3}\right)_{-}$ $\mathrm{H}$ sites that are beyond reach in conventional chain-walking events (Scheme 1b). ${ }^{7}$

Table 1. Optimization of the Reaction Conditions. ${ }^{a}$

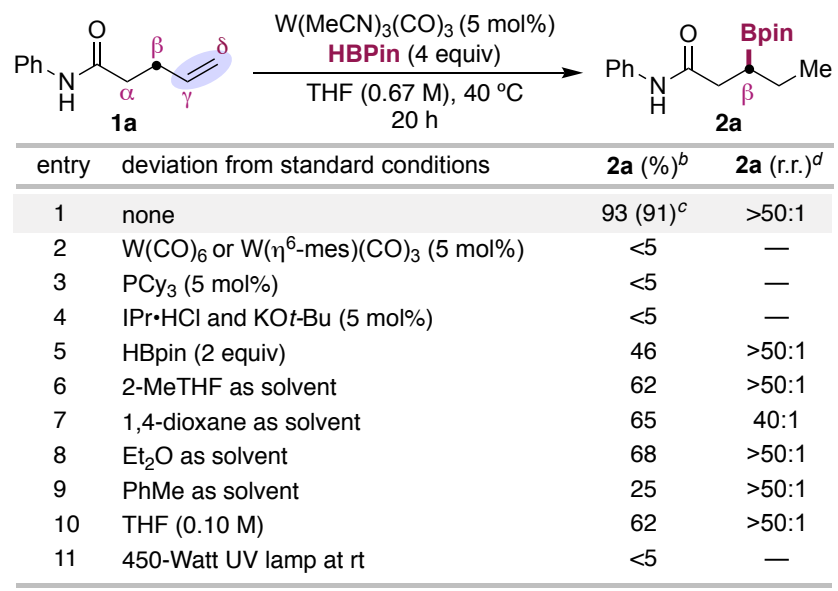

${ }^{a} \mathbf{1 a}(0.20 \mathrm{mmol}), \mathrm{W}(\mathrm{MeCN})_{3}(\mathrm{CO})_{3}(5 \mathrm{~mol} \%), \mathrm{HBPin}(0.80$ $\mathrm{mmol})$, THF $(0.67 \mathrm{M}), 40{ }^{\circ} \mathrm{C}, 20 \mathrm{~h} .{ }^{b}$ Yields determined by $\mathrm{GC}$ using decane as internal standard. ${ }^{c}$ Isolated yield. ${ }^{d}$ Regioisomeric ratio (r.r.) calculated by LCMS or GCMS between $\beta$ and $\gamma / \delta$.

Specifically, we believed that the ability of low-valent W catalysts to adopt multiple coordination geometries ${ }^{6,8-9}$ combined with their high Lewis acidity when compared to later transition metals, would be particularly critical for success. We anticipated that such a technology would not 
only expand the boundaries of chain-walking reactions by offering a complementary site-selectivity profile, but also stimulate the adoption of low-valent $\mathrm{W}$ catalysts in alkene functionalization. ${ }^{6,7 \mathrm{a}-\mathrm{c}}$ As part of our interest in alkene functionalization and chain-walking reactions, ${ }^{10}$ we describe the successful implementation of this goal, culminating in the development of the first W-catalyzed hydroboration of alkenes. ${ }^{11}$

We began our work by evaluating the catalytic borylation of 1a (Table 1). After some experimentation, ${ }^{12}$ a protocol employing commercially available $\mathrm{W}(\mathrm{MeCN})_{3}(\mathrm{CO})_{3}$ and HBpin provided the best results, delivering $\mathbf{2 a}$ as a single $\beta$-regioisomer in $91 \%$ isolated yield. As expected, the nature of the catalyst and lability of the ligands had a nonnegligible impact on reactivity. Indeed, only traces of $\mathbf{2 a}$ were observed when changing the three weakly coordi- nating $\mathrm{MeCN}$ ligands to strongly binding $\mathrm{CO}$ or $\eta^{6}$-mesitylene (entry 2). Similarly, the inclusion of phosphine ligands (entry 3 ) or the $N$-heterocyclic carbenes (entry 4) resulted in low conversions to $\mathbf{2 a}$, likely due to the formation of a catalytically inactive, more electron-rich metal center. Solvents other than THF had a deleterious impact on both yield and regioselectivity (entries 6-10). Tentatively, these observations suggest that THF might be serving as a ligand for tungsten, and/or facilitating the formation of a Lewis pair with HBpin to modulate its reactivity. As shown in entry 11, exposure of $\mathbf{1 a}$ under UV irradiation resulted in negligible formation of $\mathbf{2 a}$. Importantly, control experiments with $\beta, \gamma-$ or $\alpha, \beta$-unsaturated amides clearly show that isomerization stops at the $\beta, \gamma$-position and that hydroboration is exclusively catalyzed by tungsten. ${ }^{13}$

Table 2. Site-Selective $s p^{3}$ C-H Borylation of Unactivated Alkenes Aided by Native Directing Groups. ${ }^{a}$
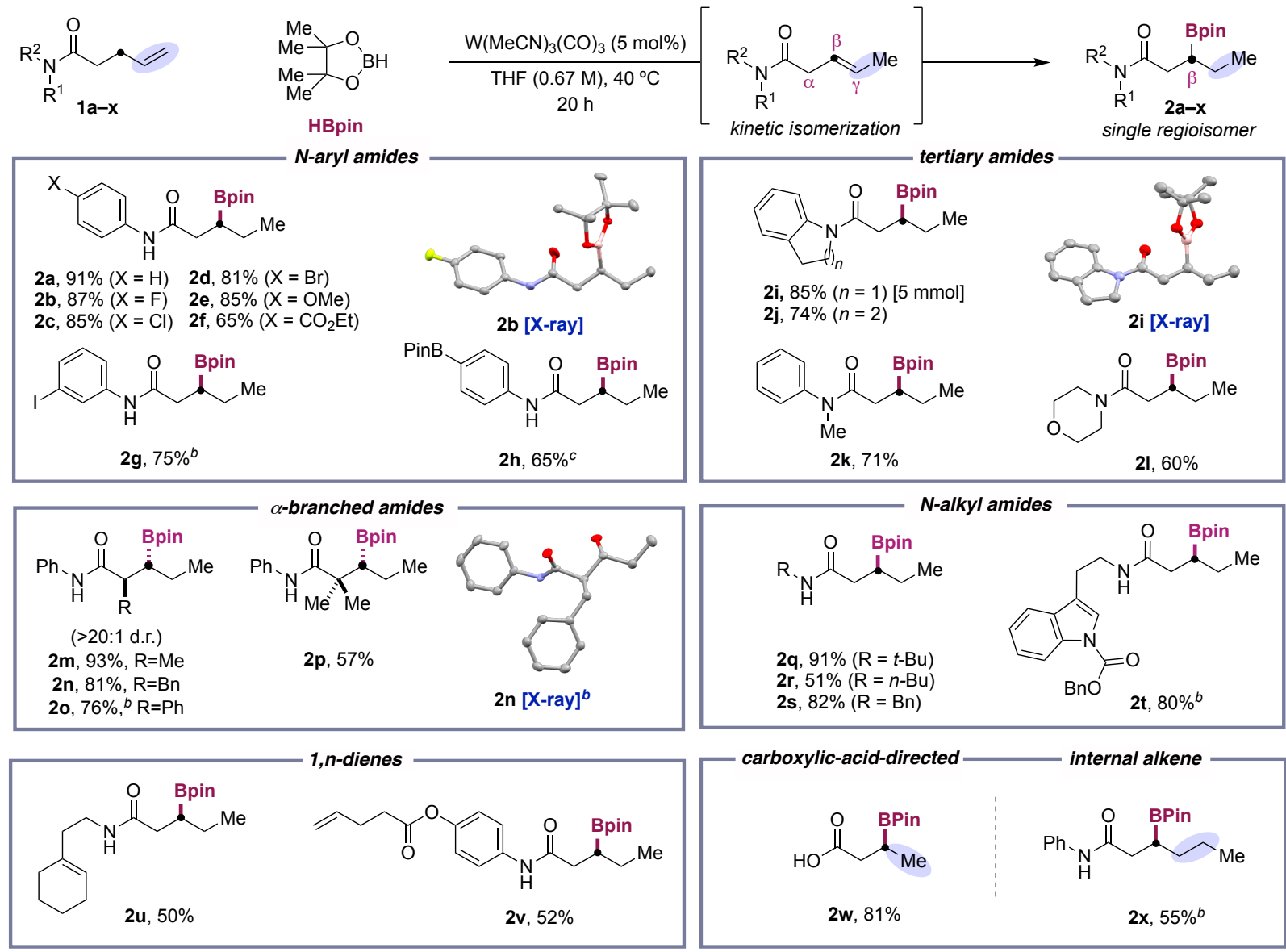

${ }^{a}$ Reaction conditions as in Table 1 , entry 1 ; Isolated yields. ${ }^{b}$ Isolated as the aliphatic alcohol following treatment with $\mathrm{H}_{2} \mathrm{O}_{2}$, aq. $\mathrm{NaOH} .{ }^{c} \mathrm{HBpin}$ (5 equiv). All depicted X-ray structures have hydrogen atoms omitted for clarity.

Next, we turned our attention to exploring the generality of our protocol. As shown in Table 2, a variety of $N$-aryl secondary amides could be employed as substrates, and the $\mathrm{C}\left(s p^{3}\right)-\mathrm{H}$ borylation could be conducted in the presence of aryl fluorides, chlorides, bromides, and iodides (2b-d, 2g). Importantly, no borylation or reduction at the
$\mathrm{C}\left(s p^{2}\right)$-halide bond was detected in the crude reaction mixtures. This finding is particularly important, showing a complementary selectivity pattern to that observed for other low-valent transition metals that would otherwise result in functionalization at the $\mathrm{C}\left(s p^{2}\right)$ site. Likewise, functional groups prone to reduction, such as esters (2f, 
$\mathbf{2 t}$ and $\mathbf{2 v}$ ), or the presence of an arylboronate (2h) did not interfere with the efficacy of the $s p^{3} \beta$-borylation event. The reaction could be extended to tertiary amides $(2 \mathbf{i}-\mathbf{l})$ and $\mathrm{N}$-alkyl secondary amides $(\mathbf{2} \mathbf{q}-\mathbf{t})$. The former observation is particularly interesting, as it does not only expand the range of amides that can be utilized, ${ }^{14}$ but also suggests that binding of the substrate to the tungsten catalyst does not require the presence of a (deprotonatable) $\mathrm{N}-\mathrm{H}$ bond. In addition, it is worth noting the reaction en route to $2 \mathbf{i}$ could be easily scaled up to $5 \mathrm{mmol}$ scale without an erosion in yield. The preparation of $\mathbf{2 i}-\mathbf{j}, \mathbf{2 l}$ and $\mathbf{2 t}$ illustrates that substrates containing $N$-heterocyclesparticularly prevalent motifs in natural products and advanced synthetic intermediates - can easily be accessed under our protocol. The chemoselectivity of our reaction is further illustrated by the successful preparation of $\mathbf{2 u}$ and $2 \mathrm{v}$ possessing additional alkenes on the side chain. Under the limits of detection, diene substrates $(\mathbf{2} \mathbf{u}, \mathbf{2 v})$ underwent monofunctionalization without additional borylation or competitive isomerization occurring at the second alkene. As shown for $\mathbf{2 w}$, the reaction could be similarly applied to internal alkenes, albeit in moderate yields. Although esters (2v) or ketones were not competent as directing groups, a free carboxylic acid delivered $\mathbf{2 w}$ as single regioisomer. Particularly noteworthy is the ability to easily access $\alpha$-branched products $\mathbf{2 m}-\mathbf{p}$ in good yields and excellent diastereoselectivities $(>20: 1)$.

Scheme 2. Preservation of an $\alpha$-Stereocenter.
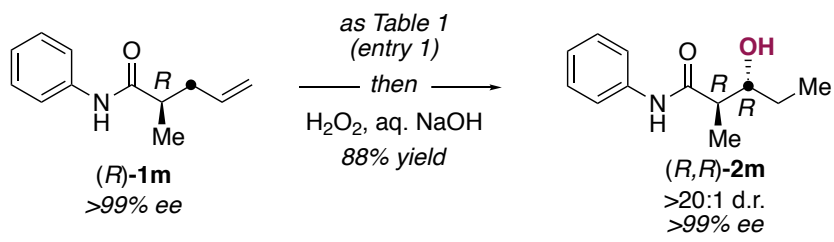

The anti-stereochemistry of substrates containing an $\alpha$ substituent was unequivocally confirmed by X-ray crystallography of the aliphatic alcohol derived from the stereoretentive oxidation of $\mathbf{2 n}$. More importantly, $\mathbf{2 m}$ could be obtained in high yield as a single enantiomer and diastereoisomer when starting from enantiopure 1m ( $>99 \%$ ee) (Scheme 2). This finding has important mechanistic implications. Specifically, it suggests that alkene isomerization stops at the $\beta, \gamma$-position, as formation of a conjugated $\alpha, \beta$-unsaturated amide would ablate the $\alpha$-stereocenter in $\mathbf{2 m}$. This observation was further corroborated by the successful preparation of $\alpha, \alpha$-gem-dimethyl substituted $2 p$ in good yield and as single regioisomer despite the proximal steric hindrance.
Scheme 3. Expansion of the Coupling Partner Scope.

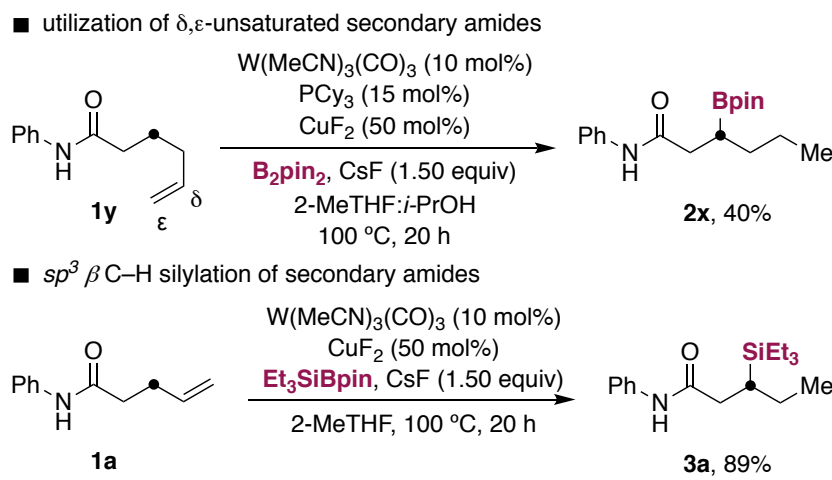

- $s p^{3} \alpha \mathrm{C}-\mathrm{H}$ germanylation of secondary amides

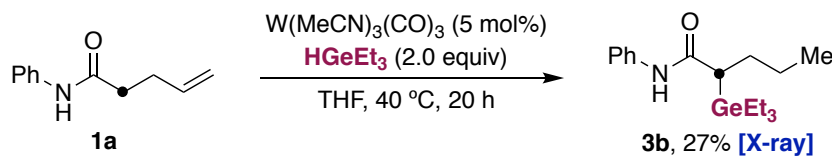

While aliphatic amides possessing a pendant alkene at the $\delta, \varepsilon$-position resulted in trace amounts of the targeted $s p^{3}$ $\beta$-borylation under the optimized reaction conditions, a protocol based on $\mathrm{CuF}_{2}$ and $\mathrm{B}_{2}$ pin 2 delivered $\mathbf{2 x}$ in moderate yield, but as a single regioisomer (Scheme 3 ). ${ }^{15} \mathrm{In}$ line with these results, we wondered whether our protocol could be extended to other C-heteroatom bond-forming reactions. Gratifyingly, this was indeed the case, and an analogous silylation event could be conducted under otherwise identical reaction conditions (3a). Intriguingly, a hydrogermanylation could also be implemented in an unoptimized $27 \%$ yield. This reaction gave rise to $\mathbf{3 b}$ with an unexpected $\alpha$-selectivity pattern, the identity of which was unambiguously determined by X-ray diffraction. While the origin of such regioselectivity remains unclear, it suggests that boranes may react differently with lowvalent tungsten catalyst when compared to other main group metal hydrides, ${ }^{8-c}$ thus setting the basis for enabling future catalytic endeavours.

\section{Scheme 4. Expansion of the Coupling Partner Scope.}

- accessing quaternary organoboranes
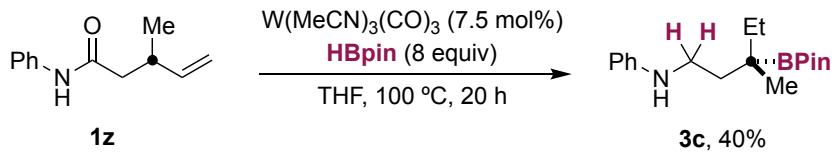

- product diversification via organotrifluoroborates
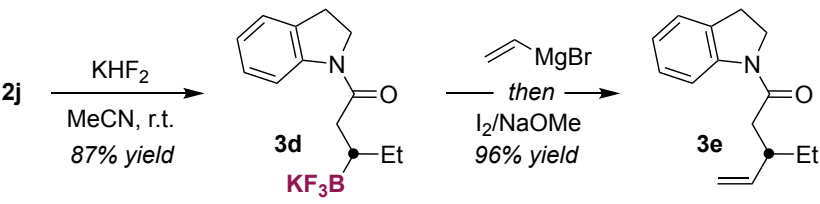

Although $\beta$-branched $\gamma, \delta$-unsaturated secondary amides showed poor yields under our optimized reaction conditions, an increase in the amount of HBpin at elevated temperatures enabled the formation of quaternary organoboranes (Scheme 4). Interestingly, a close inspection of the 
NMR data revealed in situ reduction of the carbonyl group under the reaction conditions, with $\mathbf{3 c}$ being produced in modest $40 \%$ yield. Notably, $\mathbf{2} \mathbf{j}$ can readily be converted to $\mathbf{3 d}$ by reaction with $\mathrm{KHF}_{2}$, providing an additional handle for further manipulation via cross-coupling reactions. 3d could then be further derivatized via 1,2-boronate rearrangement, affording $3 \mathbf{e}$ in $96 \%$ yield without competitive addition of the Grignard reagent to the carbonyl.

Scheme 5. Deuterium Labelling Studies.

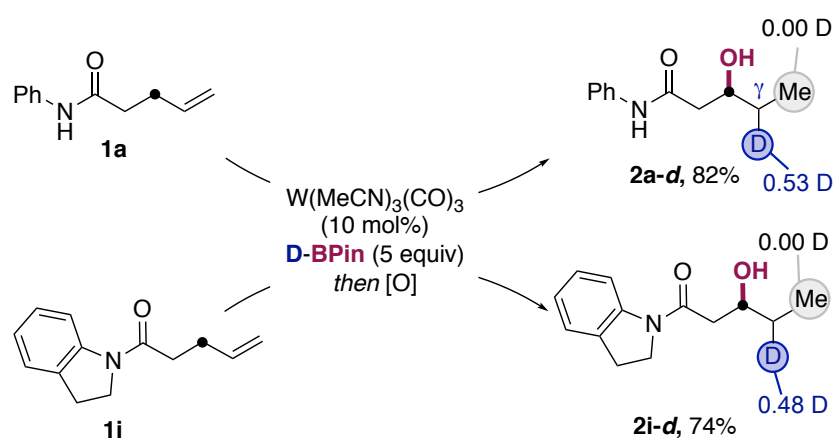

Next, we turned our attention to studying the mechanism of our $\mathrm{C}\left(s p^{3}\right) \beta$-borylation. A priori, one might expect that both 1,2- and 1,3-hydride shift might come into play for the initial alkene isomerization. ${ }^{1,16}$ To this end, we conducted the borylation of $1 \mathbf{a}$ and $\mathbf{1 i}$ with DBpin. Deuterium incorporation at the $\gamma$-position was anticipated for a pathway consisting of 1,3-hydride shift; on the contrary, labelling at the terminal $\delta s p^{3}$ site would indicate a mechanism via 1,2-hydride shift. As shown in Scheme 5, exclusive deuterium incorporation into the $\gamma$-position was observed in $\mathbf{2 a -} \boldsymbol{d}$ and $\mathbf{2} \mathbf{i}-\boldsymbol{d}$, thus strongly supporting the notion that alkene isomerization proceeds via 1,3-hydride shift. Conducting the reaction without HBpin corroborated previous findings ${ }^{16}$ that showed the ability of $\mathrm{W}(0)$ to promote isomerization via $1,3-\mathrm{H}$ shift without external hydride sources. ${ }^{12}$ In addition, the lack of deuterium incorporation at the $\beta-\mathrm{C}\left(s p^{3}\right)$ site argues against a 1,1-hydroboration of a Fischer-type W-carbene. Although other scenarios might be conceivable, ${ }^{17-18}$ we currently propose a pathway consisting of coordination of $\mathrm{W}(0)$ to both carbonyl group and the alkene (I) followed by allylic $s p^{3} \mathrm{C}-$ $\mathrm{H}$ oxidative addition (II). Re-insertion of the metal hydride to the $\pi$-allyl moiety would formally result in an olefin isomerization, thus setting the stage for an oxidative addition of $\mathrm{H}-$ Bpin to $\mathrm{W}(0)$ intermediate III (Scheme 6). Subsequently, exo-hydride insertion to the $\beta, \gamma$-alkene might form IV which ultimately undergoes $\mathrm{C}-\mathrm{B}$ reductive elimination to afford the final $s p^{3} \beta$-borylation while regenerating the propagating $\mathrm{W}(0)$ catalyst. ${ }^{18}$ From intermediate III, the isomerized alkene $\mathbf{1 a b}$ is able to reversibly dissociate, as free $\mathbf{1 a b}$ can be detected by in situ ${ }^{1} \mathrm{H}$ NMR. ${ }^{12,19}$
Scheme 6. Proposed Mechanism.

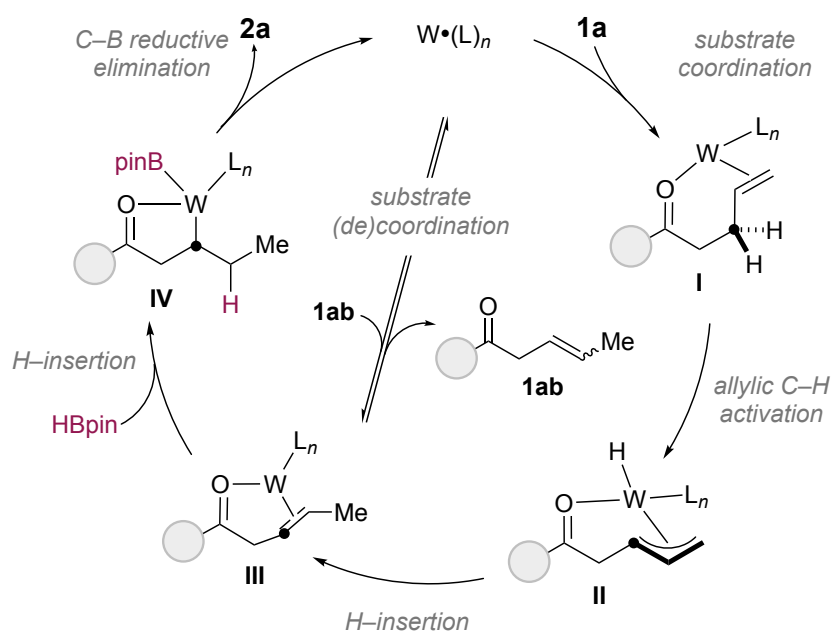

In conclusion, we have developed a protocol that illustrates the unique properties of the $\mathrm{W}(0) / \mathrm{W}$ (II) redox cycle to address site-selectivity issues that have remained challenging for other transition metals in the chain-walking arena. Our $s p^{3} \beta$-borylation is distinguished by its simplicity, mild conditions, and broad scope. This includes challenging functional group combinations, while exhibiting an exquisite chemo-, regio- and diastereoselectivity profile, thus offering a complementary technique in our synthetic repertoire for forging $s p^{3} \mathrm{C}-\mathrm{B}$ linkages.

\section{ASSOCIATED CONTENT}

\section{Supporting Information.}

The Supporting Information is available free of charge on the ACS Publications website. Experimental procedures, spectral and crystallographic data (PDF)

Crystallographic data for $\mathbf{2 b}$ (.cif)

Crystallographic data for $\mathbf{2} \mathbf{j}$ (.cif)

Crystallographic data for $\mathbf{2 u}$ (.cif)

Crystallographic data for $\mathbf{3 b}$ (.cif)

\section{AUTHOR INFORMATION}

\section{Corresponding Authors}

*rmartinromo@iciq.es; keary@scripps.edu

Author Contributions

$\S \mathrm{T}$. C. Jankins and R. Martin-Montero contributed equally to this work.

Note

The authors declare no competing financial interest.

\section{ACKNOWLEDGMENT}

We thank ICIQ, FEDER/MICIU (AEI/PGC2018-096839-B100), and MCI/AIE (Severo Ochoa Excellence Accreditation 2020-2023, CEX2019-000925-S) for financial support. R.M-M. thank La Caixa for a predoctoral fellowship. We 
thank the NIH for financial support (5R35GM125052-04). We also thank the Lindemann Trust and the English-Speaking Union for the provision of a fellowship to P. C. Dr. Milan Gembicky (UCSD) is thanked for collection and analysis of all X-ray structures. Dr. Jason Chen and Brittany Sanchez (Scripps Research Automated Synthesis Facility) are thanked for chiral separations of $\mathbf{1} \mathbf{m}$ and $\mathbf{2 m}$.

\section{REFERENCES}

1. For relevant reviews, see: (a) Vasseur, A.; Bruffaerts, J.; Marek, I. Remote Functionalization through Alkene Isomerization. Nat. Chem. 2016, 8, 209-219. (b) Sommer, H.; Juliá-Hernández, F.; Martin, R.; Marek, I. Walking Metals for Remote Functionalization. ACS Cent. Sci. 2018, 4, 153-165. (c) Fiorito, D.; Scaringi, S.; Mazet, C. Transition Metal-Catalyzed Alkene Isomerization as an Enabling Technology in Tandem, Sequential and Domino Processes. Chem. Soc. Rev. 2021, 50, 1391-1406.

2. For selected examples, see: (a) Obligacion, J. V.; Chirik, P. J., Earth-Abundent Transiton Metal Catalysts for Alkene Hydrosilylation and Hydroboration. Nat. Rev. Chem. 2018, 2, 15-34. (b) Zhang, Y.; Xu, X.; Zhu, S. Nickel-Catalysed Selective Migratory Hydrothiolation of Alkenes and Alkynes with Thiols. Nat. Comm. 2019, 10, 1752.

3. For relevant reviews on this topic, see: (a) Diaz, D. B.; Yudin, A. K., The Versatility of Boron in Biological Target Engagement. Nat. Chem. 2017, 9, 731-742. (b) Qiu, F.; Zhao, W.; Han, S.; Zhuang, X.; Lin, H.; Zhang, F. Recent Advances in Boron-Containing Conjugated Porous Polymers. Polymers 2016, 8, 191. (c) Fyfe, J. W. B.; Watson, A. J. B. Recent Developments in Organoboron Chemistry: Old Dogs, New Tricks. Chem 2017, 3, 31-55.

4. (a) Obligacion, J. V.; Chirik, P. J., Bis(imino)pyridine CobaltCatalyzed Alkene Isomerization-Hydroboration: A Strategy for Remote Hydrofunctionalization with Terminal Selectivity. J. Am. Chem. Soc. 2013, 135, 19107-19110. (b) Palmer, W N.; Diao, T.; Pappas, I.; Chirik, P. J. High Activity Cobalt Catalysts for Alkene Hydroboration with Electronically Responsive Rerpyridine and $\alpha$-Diimine Ligands. ACS Catal. 2015, 5 , 622-626. (c) Scheurmann, M. L.; Johnson, E. J.; Chirik, P. J. Alkene Isomerization-Hydroboration Promoted by Phosphine-Ligated Cobalt Catalysts. Org. Lett. 2015, 17, 27162719. (d) Léonard, N. G.; Palmer, W. N.; Friedfeld, M. R.; Bezdek, M. J.; Chirik, P. J., Remote, Diastereoselective Cobalt-Catalyzed Alkene Isomerization-Hydroboration: Access to Stereodefined 1,3-Difunctionalized Indanes. ACS Catal. 2019, 9, 9034-9044. (e) Macaulay, C. M.; Gustafson, S. J.; Fuller, J. T., III; Kwon, D.-H., Ogawa, T.; Ferguson, M. J.; McDonald, R.; Lumsden, M. D.; Bischof, S. M.; Sydora, O. L.; Ess, D. H.; Stradiotto, M.; Turculet, L. Alkene Isomerization-Hydroboration Catalyzed by First-Row Transition-Metal (Mn, Fe, Co, and $\mathrm{Ni}$ ) $N$-Phosphinoamidinate Complexes: Origin of Reactivity and Selectivity. ACS Catal. 2018, 8, 9907-9925. (f) Chen, X.; Cheng, Z.; Guo, J.; Lu, Z. Asymmetric Remote C-H Borylation of Internal Alkenes via Alkene Isomerization. Nat. Commun. 2018, 9, 3939.

5. Yu, X.; Zhao, H.; Xi, S.; Chen, Z.; Wang, X.; Wang, L.; Lin, L. Q. H.; Loh, K. P.; Koh, M. J. Site-Selective Alkene Borylation Enabled by Synergistic Hydrometallation and Borometallation. Nat. Catal. 2020, 3, 585-592.

6. Jankins, T.C.; Bell, W.C.; Zhang, Y.; Qin, Z.-Y.; Gembicky, M.; Engle, K.M. Low-Valent Tungsten Redox Catalysis Enables Controlled Isomerization and Carbonylative Functionalization of Alkenes. ChemRxiv. 2021, DOI: 10.26434/chemrxiv.14362238.
7. Examples of isomerization/ $\beta$-hydroarylation or -alkylation have been reported: (a) Ohlmann, D.K.; Gooßen, L.J.; Dierker, M. Regioselective Synthesis of $\beta$-Aryl- and $\beta$ Amino-Substituted Aliphatic Esters by Rhodium-Catalyzed Tandem Double-Bond Migration/Conjugate Addition. Chem. Eur. J. 2011, 17, 9508-9519. (b) Chen, X.; Rao, W.; Yang, T.; Koh, M.J. Alkyl Halides as Both Hydride and Alkyl Sources in Catalytic Regioselective Reductive Olefin Hydroalkylation. Nat. Comm. 2020, 11, 5857.

8. (a) Handzlik, J.; Szymańska-Buzar, T. The Formation of a $\sigma-$ Bond Complex vs. an Oxidative Addition Product in Reaction of $\left.\left[\mathrm{M}(\mathrm{CO})_{4}\left(\eta^{4}-\mathrm{nbd}\right)\right]\right](\mathrm{M}=\mathrm{W}, \mathrm{Mo})$ and $\mathrm{H}-\mathrm{EEt}_{3}(\mathrm{E}=\mathrm{Si}, \mathrm{Ge}$, Sn): DFT Optimized Structures and Predicted Chemical Shifts of Hydride Ligands. J. Organomet. Chem. 2014, 769, 136143. (b) Bożena, A.; Szymańska-Buzar, T. Photochemical Reactions of [W(CO) ${ }_{4} \eta^{4}$-nbd]] with Hydrosilanes: Generation of New Hydrido Complexes of Tungsten and Their Reactivity. $J$. Organomet. Chem. 2008, 693, 2163-2170. (c) Handzlik, J.; Kochel, A.; Szymańska-Buzar, T. H-Ge Bond Activation by Tungsten Carbonyls: An Experimental and Theoretical Study. Polyhedron 2012, 31, 622-631. (d) Hoffman, R.; Beier, B. F.; Muetterties, E. L.; Rossi, A. R. Seven-Coordination. A Molecular Orbital Exploration of Structure, Stereochemistry, and Reaction Dynamics. Inorg. Chem. 1977, 16, 511-522.

9. Trost, B. M.; Hung, M. H. Tungsten-Catalyzed Allylic Alkylations. New Avenues for Selectivity. J. Am. Chem. Soc. 1983, 105, 7757-7759.

10. For representative work, see: (a) Juliá-Hernández, F.; Moragas, T.; Cornella, J.; Martin, R. Remote Carboxylation of Halogenated Aliphatic Hydrocarbons with Carbon Dioxide. $\mathrm{Na}$ ture 2017, 545, 84-88. (b) Derosa, J. D.; Kleinmans, R.; Tran, V. T.; Karunananda, M. K.; Wisniewski, S. R.; Eastgate, M. D.; Engle, K. M. Nickel-Catalyzed 1,2-Diarylation of Simple Alkenyl Amides. J. Am. Chem. Soc. 2018, 140, 17878-17883. (c) Sun, S.-Z.; Börjesson, M.; Martin-Montero, R.; Martin, R. Site-Selective Ni-Catalyzed Reductive Coupling of $\alpha$-Haloboranes with Unactivated Olefins. J. Am. Chem. Soc. 2018, 140, 12765-12769. (d) Li, Z.-Q.; Apolinar, O.; Deng, R.; Engle, K. ChemRxiv 2021, DOI: 10.26434/chemrxiv.14639778.v1.

11. To the best of our knowledge, $\mathrm{W}$-catalyzed hydroboration has not been reported to date. For a recent perspective, see: Bage, A.D.; Nicholson, K.; Hunt, T.A.; Langer, T.; Thomas, S.P. The Hidden Role of Boranes and Borohydrides in Hydroboration Catalysis. ACS Catal. 2020, 10, 13479-13486.

12. For details, see Supporting Information.

13. The remaining mass balance corresponds to recovered starting material and a mixture of internal alkenes and reduced byproducts. See Supporting information for details.

14. Directed hydroboration of secondary or tertiary $\beta, \gamma$-alkenyl amides has been explored with varying levels regiocontrol: (a) Evan, D. A.; Fu, G. C.; Hoveyda, A. M. Rhodium(I)- and Iridium(I)-Catalyzed Hydroboration Reactions: Scope and Synthetic Applications. J. Am. Chem. Soc. 1992, 114, 6671-6679. (b) Smith, S. M.; Thacker, N. C.; Takacs, J .M. Efficient Amide-Directed Catalytic Asymmetric Hydroboration. J. Am. Chem. Soc. 2008, 130, 3734-3735. (c) Smith, S. M.; Takacs, J. M. Amide-Directed Catalytic Asymmetric Hydroboration of Trisubstituted Alkenes. J. Am. Chem. Soc. 2010, 132, 1740-1741.

15. Tentatively we believe that the $\mathrm{CuF}_{2} / \mathrm{B}_{2} \mathrm{pin}_{2}$ system may proceed through a mechanistically distinct pathway involving 1,4-protoborylation of an in situ generated $\alpha, \beta$-unsaturated amide.

16. (a) Wrighton, M.; Hammond, G. S.; Gray, H. B. Group VI Metal Carbonyl Photoassisted Isomerization of Olefins. J. Organomet. Chem. 1974, 70, 283. (b) Szymańska-Buzar, T.; Jaroszewski, M.; Wilgocki, M.; Ziółkowski, J. J. Reactivity of 
Bis(alkene) Tetracaronyl Complexes of Tungsten: Evidence for Alkene to $\pi$-Allyl Hydride Rearrangement. J. Mol. Cat. A: Chem. 1996, 112, 203.

17. At present we cannot rule out a $\mathrm{CO}$ ligand-assisted $\mathrm{H}-\mathrm{B}$ activation with low-valent tungsten catalysts. For relevant references: (a) Fuchs, J.; Irran, E.; Hrobárik, P.; Klare, H. F. T.; Oestreich, M. Si-H Bond Activation with Bullock's Cationic Tungsten(II) Catalyst: $\mathrm{CO}$ as a Cooperating Ligand. J. Am. Chem. Soc. 2019, 141, 18845. (b) Coffinet, A.; Zhang, D.; Vendier, L.; Bontemps, S.; Simonneau, A.; Borane-Catalysed Dinitrogen Borylation by 1,3-B-H Bond Addition. Dalton Trans. 2021, 50, 5582.

18. A $\sigma$-bond metathesis mechanism is unlikely as such a pathway would involve a propagating $\mathrm{W}-\mathrm{H}(\mathrm{D})$ species, which would result in deuterium incorporation at the terminal position for the reactions shown in Scheme 5. In addition, these processes are kinetically controlled, with particularly high transition state energies at secondary $\mathrm{C}\left(s p^{3}\right)$ sites. See: Thompson, $\mathrm{M}$. E.; Baxter, S. M.; Bulls, A. R.; Burger, B. J.; Nolan, M. C.; Santarsiero, B. D.; Schaefer, W. P.; Bercaw, J. E. " $\sigma-B o n d$
Metathesis" for C-H Bonds of Hydrocarbons and Sc-R ( $\mathrm{R}=$ $\mathrm{H}$, alkyl, aryl) Bonds of Permethylscandocene Derivatives. Evidence for Non involvment of the $\pi$ System in Electrophilic Activation of Aromatic and Vinylic C-H Bonds. J. Am. Chem. Soc. 1987, 109, 203. Indeed, Hartwig has showed that stoichiometric low-valent tungsten provides exclusive $\mathrm{C}-\mathrm{B}$ bondformation at the primary $\mathrm{C}\left(s p^{3}\right)$ sites: (a) Waltz, K. M.; Hartwig, J. F. Selective Functionalization of Alkanes by Transition-Metal Boryl Complexes. Science 1997, 277, 211. (b). Webster, C. E.; Fan, Y.; Hall, M. B.; Kunz, D.; Hartwig, J. F. Experimental and Computational Evidence for a Boron-Assisted $\sigma$-Bond Metathesis Pathway for Alkane Borylation. $J$. Am. Chem. Soc. 2003, 125, 858.

19. Computational work has shown that $\mathrm{H}-\mathrm{X}(\mathrm{X}=\mathrm{Si}, \mathrm{Sn}, \mathrm{Ge})$ oxidative addition to $\mathrm{W}(0)$ is slightly endergonic but kinetically accessible (ref. 7a), which is consistent with our inability to detect $\mathrm{W}$-hydrides by ${ }^{1} \mathrm{H}$ NMR spectroscopy either under our optimized reaction conditions or by reacting $\mathrm{W}(\mathrm{MeCN})_{3}(\mathrm{CO})_{3}$ with large excess of HBpin.

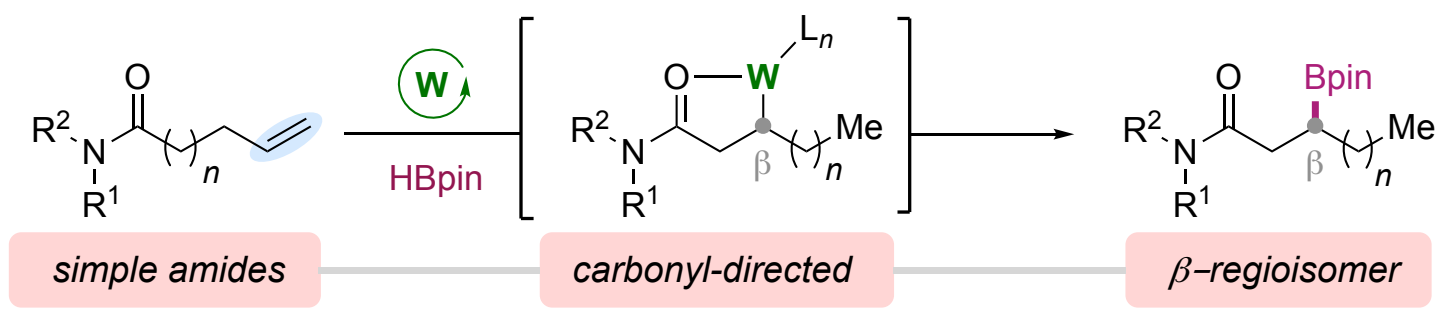

remote hydroboration $\cdot \mathrm{W}$ redox catalysis $\bullet$ unique regioselectivity $\cdot$ distinct chemoselectivity 